\title{
The prediction of benefit from pulmonary rehabilitation: setting, training intensity and the effect of selection by disability
}

\author{
M D L Morgan \\ Department of Respiratory Medicine, Glenfield Hospital, Leicester, UK
}

Introductory article

\section{Randomised controlled trial of pulmonary rehabilitation in severe chronic obstructive pulmonary disease patients, stratified with the MRC dyspnoea scale}

\section{J A Wedzicha, J C Bestall, R Garrod, R Garnham, E A Paul, P W Jones}

This study tested the hypothesis that severity of respiratory disability may affect the outcome of $\vec{\otimes}$ pulmonary rehabilitation. In this randomized, controlled study, 126 patients with chronic obstructive. pulmonary disease (COPD) were stratified for dyspnoea using the Medical Research Council (MRC) dyspnoea score into MRC3/4 (Moderate) $(n=66)$ and MRC 5 (Severe) dyspnoeic $(n=60)$ groups. The patients were randomly assigned to an eight week programme of either exercise plus education (Exercise group) or education (Control group). Education and exercise programmes for the moderately dyspnoeic $\frac{0}{0}$ patients were carried out in a hospital outpatient setting. Severely dyspnoeic patients were all treated $\overrightarrow{\vec{P}}$ at home. Those in the Exercise group received an individualized training programme. There was a 3 significant improvement in shuttle walking distance in the moderate dyspnoeic group, who received exercise training; baseline (mean \pm SEM) $191 \pm 22 \mathrm{~m}$, post-rehabilitation $279 \pm 22 \mathrm{~m}(p<0.001)$. There was no improvement in exercise performance in the severely dyspnoeic patients receiving exercise. Neither group of control patients improved. Health status, assessed by the Total Chronic Respiratory Disease $\underset{\otimes}{\ddot{x}}$ Questionnaire score, increased in the moderately dyspnoeic patients receiving exercise from $80 \pm 18$ to $95 \pm 17(p<0.0001)$ after rehabilitation. Much smaller changes were seen in the other three groups. Improvement in exercise performance and health status in patients with chronic obstructive pulmonary $\frac{\rho}{3}$ disease after an exercise programme depends on the initial degree of dyspnoea. (Eur Respir J 1998; 12:363-9)

Chronic lung disease, particularly chronic obstructive pulmonary disease (COPD), places a substantial burden of disability on sufferers and their families which conventional medical treatment can do little to alleviate. Meanwhile, enthusiastic supporters of pulmonary rehabilitation have always recognised that selective application of physical training and psychosocial support can dramatically alter the lives of some of these people with chronic lung disease. In the UK this form of treatment, aimed at reducing disability, has been slow to develop for several reasons. These may include the perception of lack of evidence for benefit as well as the absence of a culture within the speciality which acknowledges the incurable nature of many chronic lung diseases. It is certainly true that the documentary evidence for benefit has not been available in the past because of a lack of scientific interest, incentive or agreement over what to measure as benefit. There has also been no commercial or cultural pressure to develop rehabilitation services for people with lung disease and $\underset{\omega}{N}$ the government has not encouraged pulmonary rehabilitation in, for example, the same way as its cardiac $\stackrel{\varrho}{\complement}$ counterpart.

Fortunately, the status of pulmonary rehabilitation is $\stackrel{+}{+}$ now starting to improve as the evidence for benefit is beginning to accumulate. The American College of $\overrightarrow{\mathbb{D}}$ Chest Physicians and the American Association of $\frac{\mathbb{D}}{\mathbb{D}}$ Cardiovascular and Pulmonary Rehabilitation have now 응 developed evidence based guidelines which will start to standardise programmes 1 The American Thoracic Society has also just produced a new statement on pulmonary rehabilitation which summarises knowledge $\frac{\overline{0}}{2}$ to date and demystifies many of the more difficult concepts ${ }^{2}$ In addition, the British Thoracic Society is compiling a document to present the advice in a manner relevant to the National Health Service.

In this context, the paper reviewed in this article is a timely presentation of a randomised controlled trial in 
one of the areas of rehabilitation where uncertainty exists. ${ }^{3}$ It touches on many areas of interest including the assessment of disability, the measurement of outcome, and the process of physical training itself. Before appraising the paper specifically, it will be helpful to review some of these areas to place the findings of the study in the correct perspective.

\section{Assessment of disability and handicap in chronic lung disease}

The aims of pulmonary rehabilitation are to reduce symptoms, increase functional ability, and improve health status in individuals who are disabled by lung disease. An important principle in the management of this aspect of disease is the recognition that much of the resulting disability stems from the secondary effects of lung disease rather than the damage to the lung itself. Examples of these co-morbidities include the loss of skeletal muscle function, a reduction in fat free mass, and excessive exertional dyspnoea as well as less tangible deficiencies such as loss of confidence and social isolation.

With these principles in mind it is therefore reasonable to consider the assessment of patients with chronic lung disease along the lines of the WHQ classification of impairment disability and handicap 4 In this context, impairment would include reductions in static lung function, reduction in muscle strength, and in fat free mass. Disability is reflected in objective tests of exercise task performance (usually field walking tests), though this can also be inferred from structured questionnaires such as the MRC dyspnoea scale 5 Handicap is the social or physical impact of disability on the patient's life such as lack of employment. This is currently assessed by generic or disease specific health status measures which reflect the subjective health related quality of life.

There are no clear relationships between any of these classifications beyond the most general association. For example, there have been many attempts to predict exercise performance from static lung function such as forced expiratory volume in one second $\left(\mathrm{FEV}_{1}\right)$ but all have failed to identify a useful predictive relationship. Similarly, measures of handicap and disability are not congruent. This lack of agreement between measures exists because the current measurement tools are not totally specific for the area of measurement. For example, the Chronic Respiratory Questionnaire (CRQ), one of the most widely used disease specific questionnaires, covers all three elements of impairment, disability, and handicap. Also, the WHO categories do not exactly meet the needs of description in lung disease and a revision is currently underway to consider the alternative categories of impairment, activity, and participation. Other frameworks to describe the social and physical impact of lung disease - for example, the functional performance framework-also exist and have a conceptual value 7 For the present, the current WHO classifications serve well to broaden the mind in our approach to COPD and other chronic lung disease.

Within an individual a particular impairment of lung function may be associated with differing degrees of disability and handicap. When assessing a patient with lung disease prior to rehabilitation it is therefore important to do so as specifically as possible for each of the areas to be addressed. For example, it is naive to expect an improvement in $\mathrm{FEV}_{1}$ but reasonable to expect improvements in muscle strength, dyspnoea, exercise capacity, and health status.

The assessment of disability is usually done by the proxy of an exercise test. This has the appeal of simplicity and standardisation but does require some consideration. Not all exercise tests measure the same thing and may not reflect the domestic or workplace disability unless the testing circumstances are specially reproduced to mimic them. Laboratory or field exercise tests are therefore used as a practical alternative. The $\ddot{x}$ choice of exercise test requires some precision. Laborat- $\vec{\Rightarrow}$ ory tests of maximal capacity $\left(\mathrm{Vo}_{2}\right.$ peak) actually reflect $\stackrel{\mathscr{P}}{\stackrel{+}{\mathscr{C}}}$ a physiological impairment and have more value in differential diagnosis than rehabilitation assessment. 흠 Task performance tests such as constant load $\left(\% \mathrm{VO}_{2} \frac{\mathrm{G}}{\overrightarrow{\mathrm{T}}}\right.$ peak) treadmill tests are a closer reflection of disability. $\varrho$ In the UK most rehabilitation centres will not have क open access to exercise laboratories and the field exercise $\overrightarrow{0}$ test is the practical alternative. The six minute walk (6MWT) is a simple test which has served us well but $\vec{\omega}$ is not standardised and is open to the influences of mood and encouragement. The $10 \mathrm{~m}$ incremental and $\vec{x}$ endurance shuttle walk tests (ISWT and ESWT) are $₫$ the field equivalents of the laboratory maximal and 0 endurance tests. The $6 \mathrm{MWT}$ and the ISWT have be- $\infty$ come the most popular exercise tests for the assessment $\mathcal{E}$ of disability in pulmonary rehabilitation. They both 응 facilitate simple mass testing and have a sensitivity to the beneficial effects of the rehabilitation process. Tests of endurance (ESWT or constant load treadmill tests) are much more sensitive than tests of maximal capacity $\stackrel{\infty}{\oplus}$ but may be more time consuming. 10

The assessment of dyspnoea is important since it is the central symptom which rehabilitation seeks to alleviate. It is usually assessed by a visual analogue score o (VAS) or Borg scale linked to a specific exercise task such as the walking test. The degree of dyspnoea under these conditions can be modified by rehabilitation. The $\stackrel{\circ}{\varnothing}$ effect of dyspnoea on functional status can be assessed $\stackrel{\varrho}{\Rightarrow}$ bluntly by the MRC dyspnoea scale or in more detail $\frac{0}{3}$ by the dyspnoea component of the CRQ, the baseline and transitional dyspnoea indexes, and other more detailed questionnaires (UCSD, PFSDQ). . $^{-13}$

Measures of handicap are assumed to be synonymous $\overrightarrow{\vec{\partial}}$ with health status questionnaires. In fact most of the $\underset{x}{\tilde{x}}$ questionnaires cover, to a greater or lesser degree, other $\dot{\sigma}$ areas of disability. Nevertheless they offer a useful re- 3. flection of quality of life for the assessment of patients 8 undergoing pulmonary rehabilitation. General health questionnaires such as the SF36 do not have good 을 evaluative properties for the purpose of identifying bene- $>$ fit. The disease specific questionnaires such as the CRQ, 을 the St George's Questionnaire (SGQ) and the Breathing N Problems Questionnaire are more likely to change with intervention..$^{15}$ Of these instruments, the CRQ is de- O signed to be the most responsive to change and is $\omega$ therefore the most popular. Another advantage of the $\mathrm{CRQ}$ and the SGQ is knowledge of the level of change consistent with a clinically important difference. The $\mathbb{D}$ choice of health status measure is somewhat flexible since the outcomes will have a degree of dependence on the population, the context and the content of the programme.

None of the currently available outcome measures $\frac{\Omega}{\sigma}$ are perfect, or ever likely to be so, given the individual nature of goals in pulmonary rehabilitation. The overall 8 objectives of rehabilitation are to reduce disability and handicap and restore independence. It is possible that $\stackrel{\vec{O}}{\overrightarrow{7}}$ the change in exercise tests and health status measures do not correlate with improvements in domestic function or health economics. To examine these aspects it is necessary to record functional performance either directly by observation, activity monitor or, more commonly, by self report ADL questionnaire. Currently this 
sort of sophistication is not available to the average rehabilitation programme.

\section{The process and content of a pulmonary rehabilitation programme}

The aim of rehabilitation is broadly to restore the individual to the best possible level of physical and mental functioning. Some improvement is possible because the development of disability in lung disease is not caused solely by the impairment of lung function. Once dyspnoea is initiated by lung damage, disability is compounded by skeletal muscle dysfunction, poor nutrition, and psychosocial disadvantage. Rehabilitation is effective by helping these factors and should only begin when the medical management of the underlying lung disease is considered optimal.

The key components of the rehabilitation process are physical exercise training, education, and cognitive behavioural therapy. Physical training appears to be a mandatory requirement for benefit while the other activities support and augment the process. The recent evidence based guidelines set out the evidence for benefit to date for these activities in the context of formal programmes which last six weeks or more. In terms of process, lower extremity exercise training (brisk walking, cycling) is associated with strong evidence of benefit while upper body and respiratory muscle training carries a lesser strength of recommendation. In terms of benefit, rehabilitation results in a reduction in dyspnoea and improvements in quality of life. Pulmonary rehabilitation does not appear to affect survival and no good evidence yet exists for improvement in health economics. At present the evidence base is fairly small because the science of rehabilitation is in its infancy, but this is likely to expand rapidly in the future. The background issues which are pertinent to the discussion of the paper by Wedzicha et a 3 are those surrounding the selection of the patients, the setting of the programme, and the intensity of exercise training.

\section{PATIENT SELECTION}

Most papers report the benefits of rehabilitation in patients with COPD who make up the majority of those with chronic lung disease. There is growing evidence that the process is also equally effective in other conditions with similar disability. In order to benefit, patients need to be aware that they have a disability so rehabilitation is obviously inappropriate in those who only have minor symptoms where the disease has failed to make an impact. The evidence to date, which is not based on randomised controlled trials, suggests that the benefit from rehabilitation is independent of the starting level of lung function. ${ }^{16}$ In fact it appears that, in relative terms, those with a lower $\mathrm{FEV}_{1}$ seem to gain more. ${ }^{17}$ Age is also a factor which appears to be irrelevant to success and older subjects appear to tolerate and obtain benefit from the process in the same way as younger subjects. 18

The issues surrounding selection therefore relate more to the logistical difficulties of providing the service rather than identifying those who might fail to achieve benefit. There are some obvious barriers to success such as the presence of co-morbidity or poor compliance which will frustrate the process. Patients with severe cardiac disease or locomotor disorders will require a different approach. Future compliance with the rehabilitation programme is a critical requirement but difficult to ascertain. Some dropouts will be inevitable but it is obviously wasteful to take on subjects who will fail to complete the course. Identification of potential poor compliance is best left to the physician's assessment but some centres have tried to use specific questionnaires to achieve this.

Some guidance for the selection of patients for rehabilitation has been issued by the European Respiratory Society though this is not particularly directive ${ }^{10}$ It does suggest that current smokers should be excluded from $\stackrel{\oplus}{\stackrel{F}{+}}$ rehabilitation. There is no evidence to support this view and, in fact, preliminary comparisons suggest that $\frac{\overline{\bar{D}}}{\overline{\mathrm{n}}}$ smokers and ex-smokers obtain similar benefits.

SETTING

One of the principles of pulmonary rehabilitation is that it is delivered by a multi-professional team and addresses $\vec{\omega}$ the individual goals of the patients and their families. The requirements for physical training demand that this $x$ activity will be required for 3-5 sessions per week. This in turn moulds the format of the programmes which will 0 need to balance the strictures of the course requirements $\$$ with the logistics of getting patients to the programme. $\mathcal{E}$ Successful rehabilitation programmes have been de- 응 scribed in a number of settings including as a hospital inpatient, hospital outpatient, and at community centres. Hospital outpatient programmes are the most popular since they make the most efficient use of staff $\stackrel{\oplus}{\oplus}$ time. However, travel to the hospital may be difficult $\vec{\bullet}$ for severely disabled or geographically remote patients and a community based service may offer some advantages. The term "home rehabilitation" has generated some confusion in the literature since some of the successful references use the term to cover community provision or treatment at the physiotherapist's own $\stackrel{0}{\mathbb{D}}$

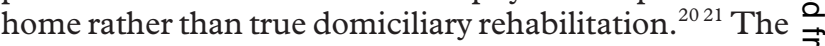
latter has never been tested. Where comparisons have been made there appears little difference, other than cost, between settings. ${ }^{22}$

\section{TRAINING INTENSITY}

It has now become the accepted view that deterioration $\stackrel{\times}{\sigma}$ in skeletal muscle function contributes to disability in 3 . COPD and other chronic lung diseases. Apart from 8 deconditioning, there may be other causes for this in- $₹$ cluding hypoxia, malnutrition, and sepsis. In healthy 윽 subjects muscle which has become weak through disuse $\rightarrow$ can be restored to vigour through the process of physical training and this is also likely to be true in patients with o lung disease. Individuals with lung disease may also gain benefits other than physiological training through lower extremity aerobic physical exercise. Physical train- $\omega$ ing can also improve confidence, exercise efficiency, and modulate dyspnoea and thereby improve performance even in the absence of a training response.

Physiological training of the skeletal muscles is a complex process which ultimately results in improved $\overline{0}$ performance (fitness) through a combination of cardiac $\mathbb{\mathbb { D }}$ adaptation, increased muscle bulk, capillarisation, and $\frac{\vec{D}}{\mathrm{D}}$ mitochondrial enzyme induction. To achieve this en- $\frac{a}{\sigma}$ hanced state in healthy subjects it is necessary to increase physical activity above the baseline in a prescribed 0 fashion. In healthy subjects the prescription for exercise is divided into the components of training intensity, frequency, and duration. To obtain a physiological training effect this stimulus should be at $50-80 \%$ of $\mathrm{Vo}_{2} \max$ for af least 20 minutes on three or four occasions each week ${ }^{23}$

In the past it has not been considered possible to obtain a physiological training response in the average 
patient with COPD. This was because it was believed that the ventilatory limit to exercise imposed by airway obstruction would prevent exercise above the gas exchange threshold and prevent the training stimulus. In addition, the only muscle biopsy study of training in COPD failed to demonstrate enzyme induction. ${ }^{24}$ Even though in pulmonary rehabilitation the traditional approach has been to train patients below the effective threshold, useful improvements have been seen, probably through non-physiological benefits.

It has recently been shown that higher intensity training can be achieved in patients with severe COPD with associated demonstration of true physiological training effects $\left.{ }^{2}\right|^{26}$ These include reductions in ventilation and lactate levels at fixed work rate and also increased oxidative enzymes in muscle biopsy specimens. Patients with COPD can sustain quite high relative workloads $\left(\% \mathrm{Vo}_{2}\right.$ peak) and high intensity $\left(60-80 \% \mathrm{Vo}_{2}\right.$ peak $)$ training is feasible. Even if patients cannot sustain this intensity, interval training at high work loads may help them achieve it eventually.

Lower extremity exercise training such as brisk walking or cycling produces improvements in functional performance which are in part due to physiological training. The benefits of the training appear to increase with the intensity, but high intensity training is not possible in all patients. Nevertheless, improvements seem to occur even at low levels and many will be unable to tolerate the highest loads. The principle should therefore be to prescribe the exercise as precisely as any other form of treatment at the highest level that the individual can manage, and constantly to review and increase that level where possible. The usual requirements for training duration and frequency are as applicable to patients as they are to healthy subjects. Currently, the details of the training programmes are poorly described in most published papers.

The other possible modes of physical training in COPD involve upper extremity, strength building, and respiratory muscle training. Some literature exists to support the value of all three modalities but these forms of physical training are task specific. Although aerobic lower limb exercise is accompanied by improvements in functional capacity, dyspnoea, and health status, this is not apparently true of the other forms of training. Improvements in upper limb or respiratory muscle strength undoubtedly occur but their relevance to the goals of the overall programme is unclear. There is some suggestion that higher intensity respiratory muscle training can augment the effects of more general aerobic training.

\section{Introductory article}

The paper by Wedzicha et a 3 adds to the growing number of randomised controlled trials of rehabilitation in COPD. The rationale for the trial was that the published improvements in exercise capacity were felt to be inconsistent and possibly related to a heterogeneity in the starting level or, alternatively, to variations in the intensity of training programmes. In this study 126 patients with COPD were randomised on the basis of their self-reported level of disability on the MRC dyspnoea scale to receive exercise training and education or education alone (control group). Rehabilitation was supervised by the same physiotherapist but was conducted in hospital for the 66 subjects in the MRC 3-4 group (have to stop after $100 \mathrm{~m}$ ) and at home for the 60 patients in the MRC 5 group (housebound). The study was designed to examine the effect of stratification by disability on the outcome of exercise capacity but the design immediately introduces the comparison of home versus hospital rehabilitation.

The active rehabilitation itself is stated to contain low intensity, unloaded upper and lower limb exercises for 으 adequate duration and frequency over eight weeks. A $\underset{x}{\mathscr{N}}$ lower limb aerobic programme included brisk walking $\vec{\Rightarrow}$ and cycling for the hospital group and brisk walking $\stackrel{\oplus}{+}$ only for the home group. The exact intensity relative to $\mathrm{Vo}_{2}$ peak is not stated although peak performance was $\frac{\bar{\sigma}}{\bar{D}}$ measured. The sessions were supervised twice weekly $\frac{\hat{\sigma}}{\vec{D}}$ and patients were encouraged to continue hourly un- $\varrho$ supervised sessions for the remaining five days. The क details of exactly how the exercise training was con- $\overrightarrow{0}$ ducted inside the patient's home may be of critical importance to the outcome.

After description of lung function, the outcome measures for this trial included exercise capacity and health $\vec{x}$ status. The measure of exercise capacity was the ISWT which was correctly conducted after one practice walk. N Health status was assessed by two disease specific ques- $\$$ tionnaires, the SGRQ and the CRQ. The authors also $\stackrel{\omega}{\infty}$ examined functional capacity with the EADL and 0 anxiety and depression with the HAD score.

The results confirmed that all groups had severe $\gg$ impairment of airway function with $\mathrm{FEV}_{1} 36-38 \%$ predicted. As expected, the $\mathrm{FEV}_{1}$ was similar in both groups $\stackrel{5}{\oplus}$ and did not relate to either reported (MRC) or measured $\vec{\theta}$ (SWT) disability. There was a substantial difference in $\underset{8}{ }$ the exercise capacity of patients with higher reported disability ( $192 \mathrm{~m}$ vs $108 \mathrm{~m}$, SWT). Interestingly, the reported disability on the MRC grade overestimates the degree of disability where patients who are supposedly housebound can manage a shuttle distance exceeding $\stackrel{\circ}{\mathbb{D}}$

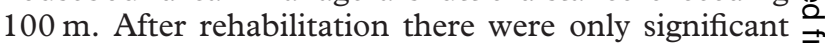
improvements in the moderately disabled group in both exercise performance and the CRQ. The SWT improved by $46 \%$ which is greater than previous general experience of about $25 \%$. The CRQ improved by a statistically and clinically significant margin but no changes $\overrightarrow{0}$ were seen in the other health status measures. This is in keeping with the general experience that the CRQ is $\dot{\sigma}$ the most sensitive questionnaire to changes following 3 rehabilitation. The other health status measures may o describe the population but are insensitive to change $₹$ following rehabilitation. The rehabilitation programme 의 had no impact on the patients with the greatest initial $>$ disability who received rehabilitation at home.

One interpretation of this study is that rehabilitation $N$ only works in patients with moderate disability in whom some function is preserved. By inference, resources may $\mathcal{N}$ be wasted on those patients with severe disability. This $\omega$ has not been the observation of previous uncontrolled studies where the benefits have been judged to be $\stackrel{0}{\subset}$ independent of the starting level of disability. This $\mathbb{D}$ requires some explanation and exposes several weaknesses with the conduct of the paper under discussion. Firstly, the authors have really tried to answer two $\mathbb{\mathbb { D }}$ questions at once by examining the effect of disability $\frac{}{\mathbb{D}}$ stratification and home versus hospital rehabilitation. 음 There are several reasons why home and hospital rehabilitation are different. Home rehabilitation lacks the peer group support of the hospital classes and also limits the access of the multi-professional team. As in this case, home subjects will also have limited access to exercise equipment. It is difficult to accept the argument that patients were too disabled to attend hospital when their objective measurement of exercise capacity should have made hospital attendance possible.

Another concern is the precision of exercise training 


\section{LEARNING POINTS}

* Pulmonary rehabilitation reduces disability and improves handicap.

* Physical training (lower limb) is an essential component of pulmonary rehabilitation.

* Exercise training should be prescribed precisely.

* Benefit increases with training intensity.

* The prior prediction of benefit following pulmonary rehabilitation is imprecise.

* There is as yet no evidence for the differential effects of various rehabilitation settings.

prescription. By the authors' description, the training target was low intensity on the basis that such severe patients were incapable of physiological training, though we now know that this is not necessarily true. Lack of confirmation of the equivalence of training intensity in the two groups leaves doubt about the adequacy of training in the home group and also prohibits any speculation about other interesting causes for the difference.

This paper is an addition to the growing number of randomised controlled trials which confirm that well structured, multi-professional, outpatient rehabilitation programmes can produce useful reductions in disability and improvements in health status. It has also reconfirmed the value of the use of appropriate outcome measures of exercise capacity and health status in assessment. Although of considerable interest, the value of selection on the basis of initial MRC dyspnoea grade remains unknown because of the failure to standardise the rehabilitation programme in terms of training prescription or setting. However, the questions remain sufficiently important and interesting enough to justify a further examination.

1 ACCP/AACPVR Pulmonary Rehabilitation Guidelines Panel, Pulmonary rehabilitation. Joint ACCP/AACVPR evidence-based guidemonary rehabilitation. Joint ACC1

2 American Thoracic Society. Statement on pulmonary rehabilitation. Am $\mathcal{F}$ Respir Crit Care Med 1999 (in press).

3 Wedzicha JA, Bestall JC, Garrod R, et al. Randomized controlled trial of pulmonary rehabilitation in severe chronic obstructive pulmonary disease patients, stratified with the MRC dyspnoea scale. Eur Respir f 1998;12:363-9.

4 World Health Organisation. International classification of impairments, disabilities and handicaps. Geneva: World Health Organisation, 1980.

5 Committee on Environmental and Occupational Health. MRC questionnaire on respiratory symptoms: instructions to interviewers. London: MRC, 1986

6 Guyatt GH, Berman LB, Townsend M, et al. A measure of quality of life for clinical trials in chronic lung disease. Thorax 1987;42:773-8.

7 Liedy NK. Functional status and the forward progress of merry-gorounds: towards a coherent analytical framework. Nurs Res 1994;43: 196-202.

8 Butland RJA, Pang J, Gross ER, et al. Two, six and twelve minute walking tests in respiratory disease. BMF 1982;284:1607-8.
9 Singh SJ, Morgan MDL, Scott S, et al. The development of a shuttle walking test of disability in patients with chronic airways obstruction. Thorax 1992;47:1019-24.

10 Revill SM, Morgan MDL, Singh SJ, et al. The endurance shuttle walk: a new field test for the assessment of endurance capacity in chronic $\bar{x}$ obstructive pulmonary disease. Thorax 1999;54:213-21.

11 Mahler DA, Wienberg DH, Wells CK, et al. The measurement of dyspnea: contents, interobserver agreement and physiological cor- iv relates of two new clinical indexes. Chest 1984;85:751-8.

12 Eakin EG, Resnikoff PM, Prewitt LM, et al. Validation of a new dyspnea $\bigcirc$ measure. The UCSD Shortness of Breath questionnaire. Chest 1998; is 113:619-24

13 Lareau SC, Meek PM, Roos PJ. Development and testing of the $\mathrm{O}$ modified version of the pulmonary functional status questionnaire (PFSDQ-M). Heart Lung 1998;27:159-68.

14 Jones PW, Quirk FH, Baveystock CM, et al. A self complete measure of health status for chronic airflow limitation. The St George's Respiratory Questionnaire. Am Rev Respir Dis 1992;145:1321-7.

15 Hyland ME, Bott J, Singh S, et al. Domains, constucts, and the development of the breathing problems questionnaire. Qual Life Res 1994;3:245-56.

16 Niederman MS, Clemente PH, Fein AM, et al. Benefits of a multi- 仓ै disciplinary pulmonary rehabilitation programme. Chest 1991;99:798804

17 ZuWallack RL, Patel K, Reardon JZ, et al. Predictors of improvement in the 12 minute walking distance following a six week outpatients pulmonary rehabilitation programme. Chest 1991;99:805-8

18 Couser JI, Guthmann R, Hamadeh MA, et al. Pulmonary rehabilitation improves exercise capacity in older elderly patients with COPD. Chest 1995;107:730-4.

19 Donner CF, Muir J. Rehabilitation and chronic care group of the European Respiratory Society. Selection criteria and programmes for pulmonary rehabilitation in COPD patients. Eur Respir f 1997;10: 744-57.

20 Wijkstra PJ, van Altens R, Kraan J, et al. Quality of life in patients with chronic obstructive pulmonary disease improves after rehabilitation in the home. Eur Respir 7 1994; 7:269-73.

21 Wiikstra PJ, van der Mark ThW, Kraan J, et al. Effects of home rehabilitation on physical performance in patients with chronic obstructive pulmonary disease. Eur Respir f 1996;9:104-10.

22 Strijbos JH, Postma DS, van Altena R, et al. A comparison between an outpatient based pulmonary rehabilitation programme and a home care pulmonary rehabilitation programme in patients with COPD. Chest 1996;109:366-72.

23 American College of Sports Medicine. Position stand. The re- O commended quantity and quality of exercise for developing and 3 maintaining cardiorespiratory and muscular fitness in healthy adults.
Med Sci Sports Exerc 1990;22:265-74.

24 Belman MJ, Kendregan BA. Physical training fails to improve ventilatory muscle endurance in patients with chronic obstructive pulmonary
disease. Chest $1982 ; 81: 440-3$.

25 Maltais F, Leblanc P, Jobin J, et al. Intensity of training and physiological $\mathrm{N}$ adaptation in patients with chronic obstructive pulmonary disease. Am 7 Respir Crit Care Med 1997;155:555-61.

26 Casaburi R, Porszasz J, Burns MR, et al. Physiologic benefits of exercise training in rehabilitation of patients with severe chronic obstructive pulmonary disease. Am f Respir Crit Care Med 1997;155:1541-51. 